\title{
Does Ambiguity Diversification Pay?
}

\author{
Yehuda Izhakian*广
}

July 24, 2012

\begin{abstract}
With a focus on risk, classical portfolio theory assumes that probabilities of future outcomes are known. In reality, however, there is ambiguity in these probabilities. This paper studies the nature of the relationship between risk and ambiguity and proves that in most cases ambiguity cannot be diversified without increasing risk. This insight implies that holding a fully diversified portfolio is not necessarily optimal. It challenges the conventional wisdom which asserts that investors should hold such a portfolio.
\end{abstract}

Keywords: Ambiguity, Ambiguity Measure, Risk, Uncertainty, Knightian Uncertainty, Random Probabilities. JEL Classification Numbers: D81, G11, G12.

*Department of Economics, Stern School of Business, New York University, yud@stern.nyu.edu

${ }^{\dagger}$ I thank Yakov Amihud, Simon Benninga, Adam Brandenburger, Itamar Drechsler, Eitan Goldman, William Greene, Sergiu Hart, Ruth Kaufman, Ilan Kremer, Yacov Oded, Efe Ok, David Schmeidler, Paul Wachtel, Avi Wohl and especially Menachem Brenner, Itzhak Gilboa, Mark Machina and Thomas Sargent for valuable discussions and suggestions. I would also like to thank the seminar participants at New York University, The Interdisciplinary Center (IDC) Herzliya, The Hebrew University of Jerusalem, University of Colorado at Boulder and Norwegian School of Business. 


\section{Does Ambiguity Diversification Pay?}

\section{Introduction}

The conventional wisdom of neoclassical portfolio theory asserts that investors should minimize risk by holding fully a diversified asset portfolio. This literature, however, assumes that probabilities of future outcomes are perfectly known, despite the fact that in reality there is ambiguity in these probabilities. ${ }^{1}$ When ambiguity is involved, two key questions arise: $(i)$ whether possessing a portfolio with minimal risk for a given expected return is optimal; $(i i)$ whether, and in what circumstances, ambiguity can be diversified away. The current paper is motivated by these questions.

The contribution of this paper to the existing literature is twofold. First, it studies the nature of the risk-ambiguity relationship and proves that ambiguity and risk are usually inversely related. To the best of our knowledge, this study is the first to address this subject. Second, it proves that in most cases risk and ambiguity cannot be simultaneously minimized by portfolio diversification.

Assuming that investors are averse to risk and to ambiguity, one might conjecture that they would aim to minimize both factors for a given expected return as they assemble their optimal asset portfolios. The compositions of these optimal portfolios are determined by the relationship between the objective risk and the objective ambiguity, as well as by the subjective level of risk aversion and the subjective level of ambiguity aversion the investors exhibit.

This paper focuses on the interplay between objective risk and objective ambiguity in isolation from investors' subjective preferences. ${ }^{2}$ To this end, it employs a measure of ambiguity that is derived form a decision-making model called expected utility with random probabilities (henceforth, EURP). This model, proposed by Izhakian (2012a), is based on Schmeidler's (1989) Choquet expected utility. Similarly to Tversky and Kahneman's (1992) cumulative prospect theory, it assumes that returns are classified either as a loss or as a gain relative to a meaningful

\footnotetext{
${ }^{1}$ Risk is defined as a condition in which the event to be realized is a-priori unknown, but the odds of all possible events are perfectly known. Ambiguity, or Knightian uncertainty, refers to conditions in which not only is the event to be realized a-priori unknown, but the odds of events are also either not uniquely assigned or are unknown.

${ }^{2}$ Previous studies focus on the relationship between attitude toward risk and attitude toward ambiguity. See, for example, Gollier (2011).
} 
reference point. ${ }^{3}$ The central concept of EURP is that not only are the returns on assets random, but the probabilities of these returns are themselves also random. Its main advantage is that, as the degree of risk can be measured by the variance of returns, so too can the degree of ambiguity be measured by the variance of the probabilities of loss (or gain). ${ }^{4}$

Ambiguity arises from random probabilities, which are assumed to be governed by the random means and random variances of normally distributed returns on assets. The degree of ambiguity is a matter of the classification of returns as losses or gains, relative to a reference point. Assuming that investors possess a reasonable reference point, the paper proves that ambiguity and risk are inversely related. Namely, when the reference point is within $\sqrt{2}$ standard deviations of the mean, ambiguity cannot be diversified without increasing risk. Perhaps counterintuitively, adding an asset to a portfolio of assets increases its ambiguity as it decreases its risk. The reason is that ambiguity is positively affected by the amplitude of the probability density function of returns. In turn, the probability density function is a negative function of variance. Higher variance results in a flatter random probability density function and a moderately sloped cumulative probability distribution. Hence, the degree of ambiguity is lower. For example, in the most extreme case, as the random variance tends to infinity, the probability density function tends to a uniform distribution, implying a zero degree of ambiguity.

The importance of ambiguity as a determinant of asset returns has been demonstrated by Brenner and Izhakian (2011), who show that ambiguity has a significant effect on the equity premium. The relevance of ambiguity for capital asset pricing has been studied by Izhakian (2012b), who proposes a generalization of the CAPM model.

The inverse relation between risk and ambiguity has considerable implications for finance. It implies that holding a fully diversified asset portfolio is not necessarily optimal for ambiguityaverse investors. This insight may shed new light on various puzzling financial phenomena. For example, individual investors tend to hold very small portfolios - only 3-4 stocks, i.e., underdiversification (see, for example, Goetzmann and Kumar (2008)), or they choose not to participate in the stock market, i.e., limited market participation (see, for example, Guo (2004) and Bogan (2008)). Another phenomenon is that expected volatility is higher than realized volatility, i.e., the volatility risk premium (see, for example, Eraker (2004), Car and Wu (2009)

\footnotetext{
${ }^{3}$ Unlike cumulative prospect theory, EURP does not assume different attitudes toward risk for losses and for gains (e.g. loss aversion).

${ }^{4}$ Measuring risk by the variance of outcomes is admissible under some conditions; the same is true for measuring ambiguity by the variance of probabilities. See Izhakian (2012a).
} 
and Drechsler (2012)). ${ }^{5}$ The relationship between risk and ambiguity can potentially be applied to further studies of these phenomena.

The main implication of our results for portfolio theory is that holding a fully diversified portfolio is usually not optimal for ambiguity-averse individuals. Accordingly, one may presume that as the degree of ambiguity associated with stocks rises, the number of stocks individuals hold in their portfolios should decline. This conclusion coincides with the findings of Guiso et al. (2008) and van Rooij et al. (2011). In their behavioral study Guiso et al. (2008) find that as individuals' faith (trust) increases, the number of stocks they hold increases, where faith is derived from the reliability of the data that individuals possess. In fact, the degree of faith is an indicator of the degree of ambiguity, which is also a matter of belief concerning the quality of information about the distribution of outcomes. Another determinant of ambiguity might be financial literacy, which is found to have a positive effect on individuals' willingness to invest in stocks (see, van Rooij et al. (2011)). Lower financial literacy implies reduced access to financial information, which in turn results in a higher degree of ambiguity.

To explain the phenomena of limited market participation and underdiversification, several theoretical studies introduce ambiguity into asset pricing models. Mukerji and Tallon (2001), for example, explain no-trading in incomplete financial markets by ambiguity aversion. Other studies that tie these phenomena to ambiguity include Dow and Werlang (1992), Epstein and Wang (1994), Trojani and Vanini (2004), Cao et al. (2005) and Ui (2011). These studies focus on individuals' reluctance to trade financial assets due to their subjective aversion to ambiguity. Unlike these studies, the current study centers around the relationship between objective risk and objective ambiguity and its implication for optimal portfolio selection. In this paper, neither preferences concerning ambiguity nor market structure (i.e., complete or incomplete) play a role.

The rest of the paper is organized as follows. Section II reviews the basic principles of the EURP model of ambiguity. Section III lays out the motivation for this study through the lens of investors' preferences. Section IV models the ambiguity of financial assets. Section V studies the risk-ambiguity relationship. Section VI discusses ambiguity diversification, and

\footnotetext{
${ }^{5}$ Other empirical phenomena referred to by the financial literature as puzzles under the rational expectations hypothesis, i.e., the assumption that there exists a unique objective probability law governing the state process and that investors know this law, include: the equity premium puzzle (Mehra and Prescott (1985)), the risk-free rate puzzle (Weil (1989)), the phenomenon wherein the observed equity volatility is too high to be justified by changes in the fundamental (Shiller (1981)), and the home bias puzzle (Coval and Moskowitz (1999)).
} 
Section VII concludes.

\section{The model of ambiguity}

The ambiguity measure derived from a model of decision making under ambiguity, called $e x$ pected utility with random probabilities (EURP), provides the theoretical underpinning of this paper. EURP, proposed by Izhakian (2012a), extends Schmeidler's (1989) Choquet expected utility by adding reference-dependent beliefs. Like Tversky and Kahneman's (1992) cumulative prospect theory, it assumes that investors have a reference point relative to which outcomes are classified as a loss or as a gain. In EURP beliefs regarding the probability of loss play an important role in measuring the degree of ambiguity. ${ }^{6}$ This theory assumes two tiers of uncertainty, one with respect to outcomes and the other with respect to the probabilities of these outcomes, each tier being modeled by a separate state space. This structure introduces a complete distinction of risk from ambiguity with regard to both beliefs and preferences. The degree of risk is then measured with respect to one space, while the degree of ambiguity is measured with respect to the second space.

Let $(\Omega, \mathcal{F}, \mathrm{P})$ be a probability space, where $\mathrm{P} \in \mathbb{P}$ is a random probability measure, and the set of probability measures $\mathbb{P}$ is closed and convex. $\mathbb{P}$ is equipped with a Borel probability measure, denoted $\chi$, with a bounded support. Given a random variable, $X: \Omega \rightarrow \mathbb{R}$, its random mean, $\mathrm{E}_{\mathrm{P}}(X)$, and random variance, $\operatorname{Var}_{\mathrm{P}}(X)$, are denoted by the Greek letters $\mu_{X}$ and $\sigma_{X}^{2}$, respectively. Similarly, the random covariance between two random variables $X$ and $Y, \mathrm{E}_{\mathrm{P}}\left(X-\mathrm{E}_{\mathrm{P}}(X)\right)\left(Y-\mathrm{E}_{\mathrm{P}}(Y)\right)$, is denoted $\sigma_{X, Y}$, and their random correlation is thus $\rho_{X, Y}=$ $\frac{\sigma_{X, Y}}{\sigma_{X} \sigma_{Y}}$.

The expectation, $\mathrm{E}[X]$, and the variance, $\operatorname{Var}[X]$, of $X$ are computed using expected probabilities. That is, a double expectation with respect to the first-order random probability distribution $\mathrm{P}$ of $X$ and to the second-order probabilities $\chi$ :

$$
\mathrm{E}[X]=\int_{\mathbb{P}}\left(\int_{\Omega} X(\omega) d \mathrm{P}(\omega)\right) d \chi(\mathrm{P})
$$

and

$$
\operatorname{Var}[X]=\int_{\mathbb{P}}\left(\int_{\Omega}(X(\omega)-\mathrm{E}[X])^{2} d \mathrm{P}(\omega)\right) d \chi(\mathrm{P})
$$

\footnotetext{
${ }^{6}$ Previous literature focuses on the implication of losses and gains for preferences (see, for example Barberis and Huang (2001) and Hirshleifer (2001)), while our study focuses on beliefs.
} 
where $\omega \in \Omega$. Similarly, the covariance between the random variables $X$ and $Y$ is given by

$$
\operatorname{Cov}[X, Y]=\int_{\mathbb{P}}\left(\int_{\Omega}(X(\omega)-\mathrm{E}[X])(Y(\omega)-\mathrm{E}[Y]) d \mathrm{P}(\omega)\right) d \chi(\mathrm{P}) .
$$

The central concept of EURP is that the probabilities of outcomes are random; thus, as the degree of risk can be measured by the variance of outcomes, the degree of ambiguity can be measured by the variance of probabilities. Let $\mathrm{P}_{L}$ and $\mathrm{P}_{G}$ be the random probabilities of loss and gain, respectively. Their expectations $\mathrm{E}\left[\mathrm{P}_{L}\right]$ and $\mathrm{E}\left[\mathrm{P}_{G}\right]$, taken with respect to the second-order probability distribution $\chi$, are

$$
\mathrm{E}\left[\mathrm{P}_{L}\right]=\int_{\mathbb{P}} \mathrm{P}(X<k) d \chi(\mathrm{P}) \quad \text { and } \quad \mathrm{E}\left[\mathrm{P}_{G}\right]=\int_{\mathbb{P}} \mathrm{P}(X>k) d \chi(\mathrm{P}),
$$

where $k$ is the reference point distinguishing losses from gains. The measure of ambiguity

$$
\mho^{2}[X]=4 \operatorname{Var}\left[\mathrm{P}_{L}\right]=4 \operatorname{Var}\left[\mathrm{P}_{G}\right]
$$

is four times the variance of the probability of loss or four times the variance of the probability of gain, taken with respect to $\chi^{7}$ This measure, $\mho^{2} \in[0,1]$, attains its minimal value, 0 , when probabilities are known, and its maximal value, 1 , only in the extreme case of a binomial probability distribution with random probabilities that can take the values 0 or 1 with equal likelihood.

To illustrate the concept of ambiguity in EURP, consider the following binomial example of an asset with two possible future returns: $d=-10 \%$ and $u=20 \%$. Assume for the moment that the probabilities of $d$ and $u$ are known, say $\mathrm{P}(d)=\mathrm{P}(u)=0.5$. The expected return is thus $5 \%$, and the standard deviation of return, measuring the degree of risk, is $15 \%$. In this case, since the probabilities are precisely known, ambiguity is not present $(\mho=0)$ and investors face only risk. Assume now that the probabilities of $d$ and $u$ can be either $\mathrm{P}(d)=0.4$ and $\mathrm{P}(u)=0.6$ or alternatively $\mathrm{P}(d)=0.6$ and $\mathrm{P}(u)=0.4$, where these two alternative distributions are equally likely. Investors now face ambiguity in addition to risk. Assuming that negative returns are considered a loss, the degree of ambiguity, measured by twice the standard deviation of the probability of loss, is $\mho=0.2$. Note that the degree of risk, computed using the expected probabilities $\mathrm{E}\left[\mathrm{P}_{d}\right]=\mathrm{E}\left[\mathrm{P}_{u}\right]=0.5$, has not changed.

\footnotetext{
${ }^{7}$ This measure is admissible for symmetric probability distributions that satisfy stochastic dominance with respect to ambiguity (see, Izhakian (2012a)).
} 


\section{Investors' preferences}

The motivation for this paper arises from an interest in understanding investors' choices regarding portfolio composition. Assuming that investors are averse to risk and to ambiguity, one might conjecture that they would aim to minimize both factors for a given expected return. To prove this we show that, for a given expected return, higher risk and higher ambiguity each result in a lower expected utility.

Preferences are the primitives of the decision-making model we employ to form expected utility. Aversion to ambiguity is exhibited when an investor prefers the expectation of the random probability of each outcome over the random probability itself. These preferences concerning ambiguity are modeled by a strictly-increasing, continuous and twice-differentiable function $\Gamma:[0,1] \rightarrow \mathbb{R}$, called the outlook function. Ambiguity aversion takes the form of a concave $\Gamma$, while ambiguity loving takes the form of a convex $\Gamma$, and ambiguity neutrality the form of a linear $\Gamma$. Preferences concerning risk are modeled by a strictly-increasing, continuous and twice-differentiable utility function $\mathrm{U}: \mathbb{R} \rightarrow \mathbb{R}$, which is normalized to $\mathrm{U}(1+k)=0$. As usual, risk aversion takes the form of a concave U, risk loving the form of a convex $\mathrm{U}$, and risk neutrality the form of a linear $\mathrm{U}$.

As a consequence of the nonlinear ways in which individuals may interpret random probabilities, perceived probabilities are nonadditive. Ambiguity aversion results in a subadditive probability measure, while ambiguity loving results in a superadditive measure. ${ }^{8}$ Formally, in this framework, the expected utility of saving one unit of wealth takes the form

$$
\begin{aligned}
\mathrm{V}(1+X)= & -\int_{-\infty}^{k} \Gamma^{-1}\left(\int_{\mathbb{P}} \Gamma(\mathrm{P}(\mathrm{U}(1+X)<z)) d \chi(\mathrm{P})\right) d z \\
& +\int_{k}^{\infty} \Gamma^{-1}\left(\int_{\mathbb{P}} \Gamma(\mathrm{P}(\mathrm{U}(1+X)>z)) d \chi(\mathrm{P})\right) d z
\end{aligned}
$$

where $X$ is the return on investment. The value function $\mathrm{V}$, proposed by Izhakian (2012a), is based on the functional representation of Wakker (2010) and Kothiyal et al. (2011). This function applies a two-sided Choquet integration to gains and to losses (relative to the reference point). Note that when investors are ambiguity neutral, i.e., $\Gamma$ is linear, Equation (6) collapses to the conventional expected utility.

\footnotetext{
${ }^{8}$ Nonadditivity means that probabilities do not necessarily add up to 1.
} 
Theorem 1. Consider an asset whose return, $X$, is relatively small with relatively small probabilities. Assuming a risk and ambiguity averse investor whose reference point $k$ is relatively close to $\mathrm{E}[X]$, higher risk and higher ambiguity each independently result in a lower expected utility.

This theorem proves that, for a given expected return, a rational (risk and ambiguity averse) investor prefers the portfolio with the lower risk and the lower ambiguity. This brings us to inquire whether risk and ambiguity can be minimized simultaneously.

\section{Ambiguity in financial assets}

The return on an asset takes the form of a random variable, $X$, with a non-unique probability distribution. ${ }^{9}$ A return, denoted $x$, is assumed to be normally distributed with a random mean and a random variance. ${ }^{10}$

The probabilities of loss are measured with respect to a reference point $k$, which is assumed to satisfy $0 \leq k \leq \mathrm{E}[X]$. Otherwise, if $k<0$, negative outcomes (returns) are considered a gain. On the other hand, if $\mathrm{E}[X]<k$, the expected return $\mathrm{E}[X]$ is considered a loss and thus investors will not hold the asset. Since returns are normally distributed, probabilities of loss can be defined as a function of $\mu_{X}$ and $\sigma_{X}$. That is,

$$
\mathrm{P}(x \leq k)=\int_{-\infty}^{k} \frac{1}{\sqrt{2 \pi \sigma_{X}^{2}}} e^{-\frac{\left(x-\mu_{X}\right)^{2}}{2 \sigma_{X}^{2}}} d x .
$$

The measure of ambiguity, $\mho^{2}$, can then be established using the standard normal probability distribution, denoted $\Phi$. Hence, $\mho^{2}$ takes the form ${ }^{11}$

$$
\mho^{2}[X]=4 \operatorname{Var}\left[\int_{-\infty}^{\frac{k-\mu_{X}}{\sigma_{X}}} \frac{1}{\sqrt{2 \pi}} e^{-\frac{x^{2}}{2}} d x\right]=4 \operatorname{Var}\left[\Phi\left(\frac{k-\mu_{X}}{\sigma_{X}}\right)\right],
$$

where the (random) probability density function is defined by $\phi\left(\frac{x-\mu_{X}}{\sigma_{X}}\right)=\frac{1}{\sqrt{2 \pi \sigma_{X}^{2}}} e^{-\frac{\left(x-\mu_{X}\right)^{2}}{2 \sigma_{X}^{2}}}$.

\footnotetext{
${ }^{9} \mathrm{An}$ individual asset or an asset portfolio is referred to by its return, $X$.

${ }^{10}$ This probability density function implies that returns satisfy first-order stochastic dominance with respect to ambiguity (see Izhakian (2012a)).

${ }^{11}$ This representation is obtained by changing the integration variable of the normal probability distribution.
} 
Proposition 1. The degree of ambiguity of an asset remains unchanged when the same monotonic transformation is applied to its return and to the reference point. That is,

$$
\mho^{2}[\mathrm{~T}(X)]=\mho^{2}[X]
$$

for any monotonic function $\mathrm{T}$.

This proposition asserts that ambiguity is invariant to monotonic transformations of returns as long as the reference point is adjusted accordingly. The reason is that ambiguity does not depend upon cardinal values but only upon ordinal values, relative to the reference point. A monotonic transformation of returns, accompanied by an identical transformation of the reference point, does not affect the ordinal values relative to the reference point. Thus, neither cumulative probability of loss nor ambiguity are changed.

Corollary 1. Applying an affine transformation, $c+b x$, to an asset return and to the reference point $k$ leaves the degree of ambiguity unchanged. That is,

$$
\mho^{2}[c+b X]=\mho^{2}[X]=4 \operatorname{Var}\left[\int_{-\infty}^{k} \frac{1}{\sqrt{2 \pi \sigma_{X}^{2}}} e^{-\frac{\left(x-\mu_{X}\right)^{2}}{2 \sigma_{X}^{2}}} d x\right] .
$$

The random parameters, $\mu_{X}$ and $\sigma_{X}$, which govern probabilities and ambiguity also characterize the expected return (perceived mean) and the risk (perceived volatility) associated with an asset. The next proposition ties the perceived mean and the perceived volatility, defined by Equations (1) and (2), to the random mean and variance.

Proposition 2. The perceived mean is equal to the expected mean, but the perceived volatility $\operatorname{Var}[X]$ is always greater than the expected variance. That is,

$$
\mathrm{E}[X]=\mathrm{E}\left[\mu_{X}\right] \quad \text { and } \quad \operatorname{Var}[X]=\mathrm{E}\left[\sigma_{X}^{2}\right]+\operatorname{Var}\left[\mu_{X}\right],
$$

where the expectation and the variance on the right-hand sides of the equations are taken with respect to the second-order probability distribution $\chi$.

This proposition implies that a-priori the perceived volatility is higher than the simple expected variance. This insight might provide a possible explanation for the phenomenon known in the financial literature as the volatility (risk) premium (see, for example, Broadie et al. (2009), Bollerslev et al. (2009), Todorov (2010) and Drechsler (2012)). The volatility premium can be identified by the difference between the volatility implied by VIX and the realized volatility in the stock market. The former can be considered a proxy for the perceived volatility, and the latter can be considered a proxy for the expected variance. Thus, Proposition 2 suggests 
that the volatility premium can be explained by the difference between perceived volatility and average variance, namely, by $\operatorname{Var}\left[\mu_{X}\right]$.

\section{The risk-ambiguity relationship}

The common thread between risk and ambiguity is the random variance $\sigma_{X}^{2}$. In order to maintain focus on the risk-ambiguity relationship, it is assumed that changes in $\sigma_{X}^{2}$ do not affect the random mean $\mu_{X}$. Since the variance $\sigma_{X}^{2}$ is random, a higher variance is obtained by applying a monotonic increasing transformation to the entire distribution of $\sigma_{X}^{2}$ such that $\mu_{X}$ remains unchanged. As a result, by Proposition 2, the higher $\sigma_{X}^{2}$, the higher the risk (perceived volatility) $\operatorname{Var}[X]$.

The random variance $\sigma_{X}^{2}$ also affects the degree of ambiguity. Ambiguity is a function of cumulative probabilities, defined by a probability density function, which in turn is a function of $\sigma_{X}^{2}$. Generally speaking, a higher $\sigma_{X}^{2}$ implies a flatter (random) probability density function, which in turn implies a lower ambiguity. Take, for example, the most extreme case where the variance tends to infinity. The probability distribution tends to a uniform distribution, which results in a zero degree of ambiguity. This can also be observed from Equation (8). As the variance tends to infinity, the probability of loss, $\Phi\left(\frac{k-\mu_{X}}{\sigma_{X}}\right)$, tends to $\frac{1}{2}$ regardless of the reference point $k$ and the mean $\mu_{X}$. Hence, the variance of the probability of loss is 0 , and so too is the degree of ambiguity.

The domain of the probability distributions can be divided into two subdomains. One is referred to as the negative domain, in which a higher variance implies lower probabilities. The other is referred to as the positive domain, in which a higher variance implies higher probabilities. $^{12}$ The inclusion of the reference point in one of these domains determines the relationship between ambiguity and risk. Namely, if the reference point is within the negative domain, ambiguity and risk are negatively related. If the reference point is within the positive domain, they are positively related. These two domains are defined by the derivative of the probability of loss, $\Phi\left(\frac{k-\mu_{X}}{\sigma_{X}}\right)$, with respect to the standard deviation $\sigma_{X}$, which in turn is defined by the derivative of the probability density function, $\frac{\partial \phi}{\partial \sigma_{X}}$. The negative domain is characterized by a decreasing derivative, $\frac{\partial^{2} \phi}{\partial \sigma_{X}^{2}}<0$, and the positive domain is characterized by an increasing derivative, $\frac{\partial^{2} \phi}{\partial \sigma_{X}^{2}}>0$. The following theorem uses this notion to define the

\footnotetext{
${ }^{12}$ The positive domain consists of two disjoint subsets of the reals.
} 
conditions under which a higher variance leads to a lower degree of ambiguity.

Theorem 2. Assume an asset whose return is normally distributed with random mean, $\mu_{X}$, and random variance, $\sigma_{X}^{2}$, and a reference point $k$ satisfying

$$
\mu_{X}-\sigma_{X} \sqrt{2} \leq k \leq \mu_{X}+\sigma_{X} \sqrt{2}
$$

for every $\mu_{X}$ and $\sigma_{X}$. A higher (random) variance implies a lower degree of ambiguity.

A reasonable reference point would be lower than (but relatively close to) the expected return on an asset and higher than the return on a risk-free asset. Otherwise, the investor would not hold such an asset. Therefore, in most cases Equation (11) holds.

As the variance decreases, the probability density function becomes steeper. Theorem 2 implies that as a result, when $k$ is in the interval $\left[\max \left(\mu_{X}-\sigma_{X} \sqrt{2}\right), \min \left(\mu_{X}+\sigma_{X} \sqrt{2}\right)\right]$, the variance of the probability of loss is higher, and therefore the degree of ambiguity is higher. However, decreasing the variance to a level lower than $\frac{\left(k-\mu_{X}\right)^{2}}{2}$ (while keeping all other parameters unchanged) causes $k$ to deviate from the interval $\left[\max \left(\mu_{X}-\sigma_{X} \sqrt{2}\right), \min \left(\mu_{X}+\sigma_{X} \sqrt{2}\right)\right]$, which is now smaller. In this case, a lower variance results in a lower degree of ambiguity.

When the variance tends to zero, the degree of ambiguity tends to zero, unless the reference point is surrounded by the random mean, wherein the degree of ambiguity tends to one. If the mean is known, i.e., the expected return is identical for all feasible distributions, and it is equal to the reference point, then the degree of ambiguity is zero regardless of the variance. ${ }^{13}$ If, however, the reference point is not equal to the common mean, then a strictly positive degree of ambiguity is observed.

Figure 1 depicts the impact of the random standard deviation, $\sigma_{X}$, on the degree of ambiguity. It considers a normally distributed return with known mean $\mu_{X}=0.1$; random standard deviation that can be either $\sigma_{X, 1}=0.05$ or $\sigma_{X, 2}=0.1$ with equal likelihoods; and reference point $k=0.09$. The $\mathrm{y}$-axis describes the degree of ambiguity, and the $\mathrm{x}$-axis describes an incremental factor $\lambda$ of the standard deviation. Namely, for a given value of $\lambda$, the standard deviation can be either $\lambda \sigma_{X, 1}$ or $\lambda \sigma_{X, 2}$. This figure shows that only for a narrow range of $\lambda$, between 0 and 0.14 , a higher standard deviation results in a higher degree of ambiguity. When $\lambda=0.14$ the standard deviation can be either 0.007 or 0.014 . In this case, since $k=0.09$, Equation (11) is violated, and so ambiguity and risk are positively related. For any $\lambda$ greater

\footnotetext{
${ }^{13}$ Even if the instances of the random probability distribution take different functional forms, the degree of ambiguity is zero as long as all distributions are symmetric with an identical point of symmetry which is equal to the reference point.
} 
than 0.14 Equation (11) holds such that a higher variance implies a lower degree of ambiguity.

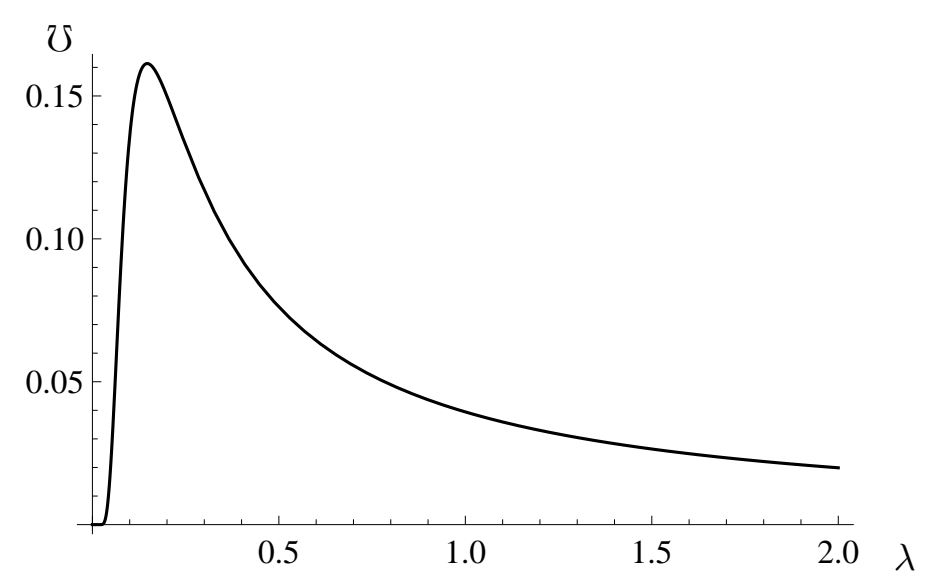

Figure 1: Ambiguity and risk

This figure describes the effect of increasing (random) standard deviation by factor $\lambda$ on the degree of ambiguity $\mho$. It considers a normally distributed return with known mean $\mu_{X}=0.1$; random standard deviation that can be either $\sigma_{X, 1}=0.05$ or $\sigma_{X, 2}=0.1$ with equal likelihoods; and reference point $k=0.09$. The $\mathrm{y}$-axis describes the degree of ambiguity, and the $\mathrm{x}$-axis describes the incremental factor $\lambda$. For a given value of $\lambda$ the standard deviation can be either $0.05 \lambda$ or $0.1 \lambda$. A higher $\lambda$ implies a higher risk.

A high degree of ambiguity is caused by high probability perturbations, derived from the random mean, $\mu_{X}$, and the random variance, $\sigma_{X}^{2}$. Figure 2 illustrates the interplay between the random variance and the random probability of loss in a standard normal probability representation. Panel I describes the probability density function, while Panel II describes its associated cumulative probability distribution. The values on the x-axes are the upper integration limits, $\frac{k-\mu_{X}}{\sigma_{X}}$, of the probability of loss, i.e., the adjusted reference point $k$ (see Equation (8)). The y-axis in Panel I describes the amplitude of the probability density function, and the y-axis in Panel II describes the cumulative probability of loss. The light shaded area depicts the range in which the integration limit, $\frac{k-\mu_{X}}{\sigma_{X}}$, is volatile when the random variance of return is relatively low, and the dark area depicts the range when it is relatively high.

Panel II of Figure 2 shows that a lower variance of return shifts the region in which the probability of loss is volatile from a range of steeper cumulative probability distribution to a range of flatter cumulative probability distribution. This implies a positive effect of variance on the degree of ambiguity. However, lower variance has a second and opposite effect on ambiguity: the lower the variance, the higher the dispersion of the random probability of loss. When the reference point $k$ satisfies $-1 \leq \frac{k-\mu_{X}}{\sigma_{X} \sqrt{2}} \leq 1$, the dispersion effect is stronger than the shifting effect, resulting in a higher degree of ambiguity. When $k$ violates $-1 \leq \frac{k-\mu_{X}}{\sigma_{X} \sqrt{2}} \leq 1$, the cumulative probability distribution is sufficiently flat such that the shifting effect is stronger 


\section{Panel I: Probability density function}

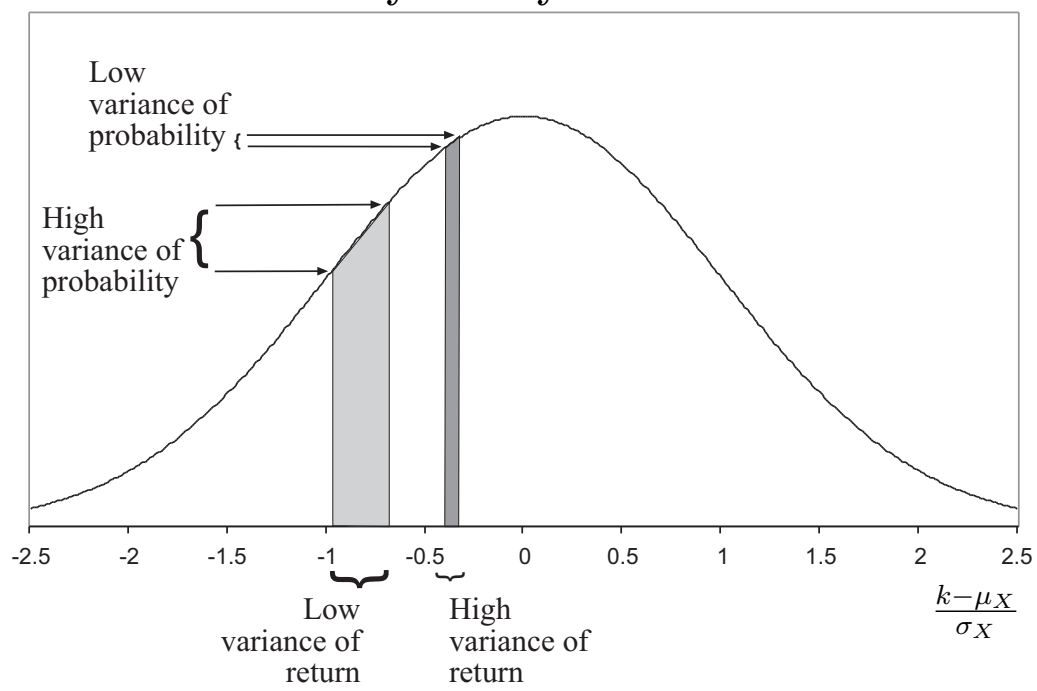

\section{Panel II: Cumulative probability distribution}

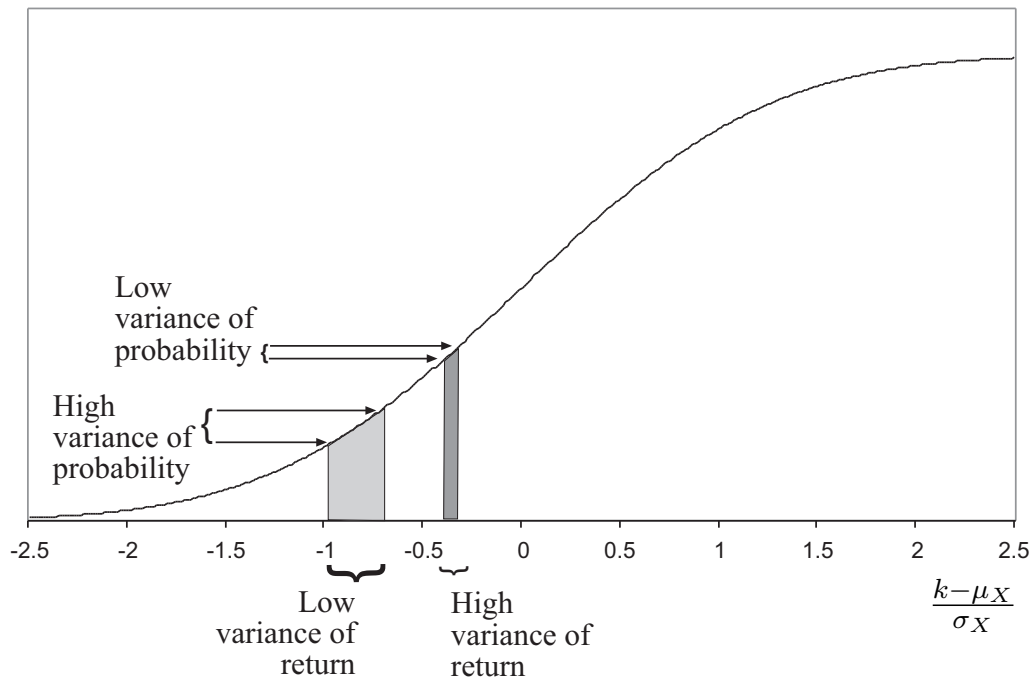

Figure 2: Random probability of loss in a standard normal probability representation

Using standard normal probability representation, this figure describes the region in which the random probability of loss is volatile. Panel I describes the probability density function, and Panel II describes its associated cumulative probabilities. The values on the $\mathrm{x}$-axes are the upper integration limit $\frac{k-\mu_{X}}{\sigma_{X}}$ of the probability of loss, i.e., the adjusted reference point $k$. The y-axis in Panel I describes the amplitude of the probability density function, and the y-axis in Panel II describes the cumulative probability. The light shaded region depicts the area where $\frac{k-\mu_{X}}{\sigma_{X}}$ is volatile when the random variance of return is relatively low, and the dark area depict the range when it is relatively high.

than the dispersion effect, resulting in a lower degree of ambiguity.

Figure 3 provides an additional view of the interplay between the random variance of return and the random probability of loss. It considers the probability density function of an asset whose mean return is known and whose variance is random. Each shaded area describes a 
range in which the probability density function is volatile as a result of the random variance. The light shaded area depicts this range when the random variance of return is relatively low and the dark area when it is relatively high. Figure 3 shows that when the reference point is relatively close to the mean, a lower variance of return (depicted by the light area) results in a sharper curve. In this case, a sharper random density function positively affects the variance of probabilities and thus the degree of ambiguity.

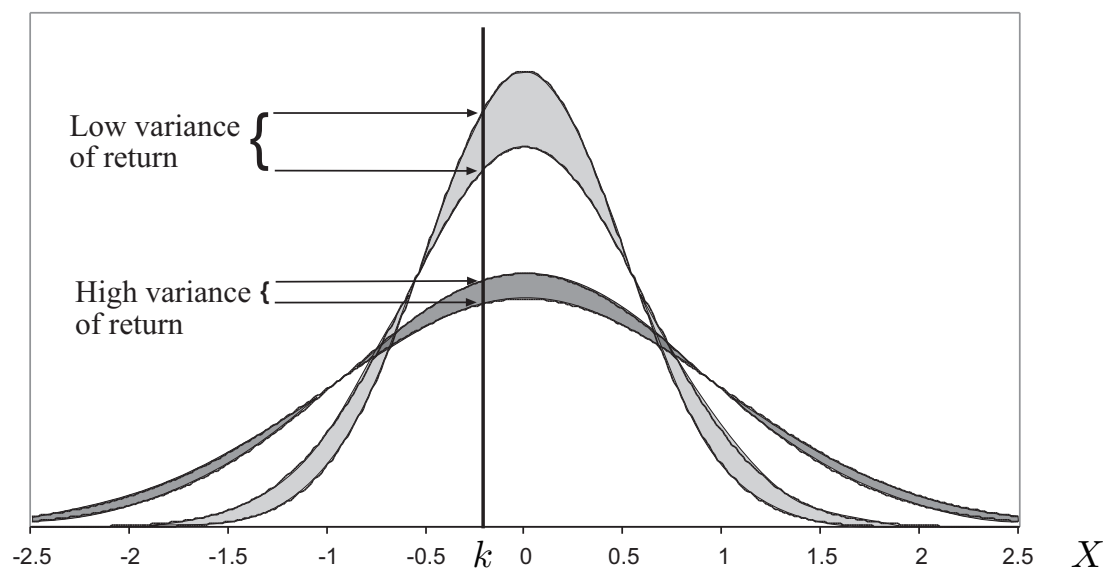

Figure 3: Random probabilities of a normally distributed return with known mean and unknown variance

This figure describes the random probability density function of a normally distributed return with known mean and unknown variance. Each shaded area describes a range in which the random probability density is volatile. The light shaded area depicts this range when the random variance of return is relatively low and the dark area when it is relatively high.

\section{Ambiguity diversification}

Can ambiguity be diversified away in an asset portfolio? In other words, are two assets less ambiguous when combined than each asset separately? Motivated by these questions, let us begin with an illustration.

Assume two assets, $X$ and $Y$, with three states of nature. The returns on $X$ and $Y$ (in percentage points) are $X=(-2,1,2)$ and $Y=(2,1,-2)$, respectively. The possible probabilities of the three states of nature are

$$
\left[\begin{array}{l}
\mathrm{P}_{1} \\
\mathrm{P}_{2}
\end{array}\right]=\left[\begin{array}{lll}
0.1 & 0.8 & 0.1 \\
0.2 & 0.6 & 0.2
\end{array}\right]
$$

where a row, indexed $i=1,2$, is a probability distribution, and a column, indexed $j=1,2,3$, 
is a state of nature. The probability distributions $\mathrm{P}_{1}$ and $\mathrm{P}_{2}$ are assigned with equal (secondorder) probabilities, i.e., $\chi=(0.5,0.5)$. The expected probabilities of each state of nature are therefore

$$
\mathrm{E}[\mathrm{P}]=\chi_{1} \mathrm{P}_{1}+\chi_{2} \mathrm{P}_{2}=(0.15,0.7,0.15)
$$

Using the expected probabilities, $\mathrm{E}[\mathrm{P}]$, one can compute the perceived mean (expected return) and the perceived volatility (risk). The expected return on assets $X$ and $Y$ is identical, $\mathrm{E}[X]=$ $\mathrm{E}[Y]=0.7$, and their risk is also identical, $\operatorname{Var}[X]=\operatorname{Var}[Y]=1.41$.

To compute the degree of ambiguity associated with each asset, the set of loss events first needs to be identified. Assuming a reference point $k=0.5$, asset $X$ faces a loss in state $j=1$, and asset $Y$ faces a loss in state $j=3$,

$$
\begin{aligned}
& \begin{array}{ll}
X & Y
\end{array} \\
& {[\underbrace{-2}_{L} \mid \underbrace{1 \quad 2}_{G}] \quad[\underbrace{21}_{G} \mid \underbrace{-2}_{L}] .}
\end{aligned}
$$

Conditional upon the probability measures $\mathrm{P}_{1}$ and $\mathrm{P}_{2}$, the probabilities of loss on asset $X$ are $\mathrm{P}_{X, L}=(0.1,0.2)$, and the same for asset $Y, \mathrm{P}_{Y, L}=(0.1,0.2)$. The variance of the probability of loss on each of these assets is identical, $\operatorname{Var}\left[\mathrm{P}_{X, L}\right]=\operatorname{Var}\left[\mathrm{P}_{Y, L}\right]=0.0025$, which implies also an identical degree of ambiguity, $\mho[X]=\mho[Y]=0.1$.

Consider now an equally weighted portfolio, denoted $Z$, consisting of assets $X$ and $Y$. Conditional upon the state of nature, the return on this portfolio is

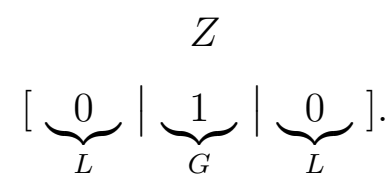

Using the expected probabilities, $\mathrm{E}[\mathrm{P}]$, to compute the expected return and the risk of portfolio $Z$ provides $\mathrm{E}[Z]=0.7$ and $\operatorname{Var}[Z]=0.21$, respectively. Portfolio $Z$ faces a loss in states $j=$ 1,3 ; therefore, its probabilities of loss $\mathrm{P}_{Z, L}=(0.2,0.4)$ imply a degree of ambiguity $\mho[Z]=0.2$. Portfolio $Z$ maintains the same expected return as assets $X$ and $Y, \mathrm{E}[Z]=\mathrm{E}[X]=\mathrm{E}[Y]=0.7$, but with lower risk, $0.21=\operatorname{Var}[Z]<\operatorname{Var}[X]=\operatorname{Var}[Y]=1.41$. Its degree of ambiguity, however, is doubled $\mho[Z]=2 \mho[X]=2 \mho[Y]=0.2$, showing that the degree of ambiguity of two assets can be greater when considered together in a portfolio than the degree of ambiguity of each asset when considered separately.

Assuming that short selling is prohibited, this example demonstrates a case where risk can be diversified away but ambiguity cannot. Even if short selling were allowed in this ex- 
ample, ambiguity still could not be diversified without increasing risk. Conditional upon the reference point, there is generally a tradeoff between risk and ambiguity, such that ambiguity diversification cannot be achieved without a higher exposure to risk. We now turn to prove it theoretically.

Assume a portfolio $Z$ consisting of $n$ assets, indexed $j=1, \ldots, n$. The return on this portfolio is thus

$$
Z=\sum_{j=1}^{n} h_{j} X_{j}
$$

where $h_{j}$ is the proportion of asset $j$ having a return $X_{j}$, such that $\sum_{j} h_{j}=1 .{ }^{14}$ The reference point $k$ is assumed to be a weighted average of the reference points associated with the assets composing the portfolio; that is, $k=\sum_{j} h_{j} k_{j}$. The probabilities of loss and gain on portfolio $Z$ are determined by loss events and gain events relative to $k$. It is important to note that the random probabilities of loss on individual assets are not independent across assets. Hence, the probability of loss on an asset portfolio is also determined by the correlations among the probabilities of loss on the individual assets composing it.

To account for the correlations among the probabilities of loss on individual assets, the probability of loss on an asset portfolio is represented by a convolution of the probabilities of the assets composing it. Assume, for example, a portfolio consisting of two assets $X$ and $Y$ with proportion $h_{1}$ and $h_{2}$, respectively. Its degree of ambiguity can be defined by the convolution

$$
\mho^{2}\left[h_{1} X+h_{2} Y\right]=2 \operatorname{Var}\left[\int_{-\infty}^{k} \int_{-\infty}^{\infty} f(x, y) d y d x\right]
$$

where $f(x, y)$ is the (random) joint probability density of the random variables $X$ and $Y$. Concerning assets whose returns are normally distributed, Equation (13) can be evolved to a closed-form solution for the degree of ambiguity associated with an asset portfolio.

Theorem 3. Let portfolio $Z$ consist of $n$ assets whose returns are normally distributed with random means, $\mu_{X_{1}}, \ldots, \mu_{X_{n}}$, and random standard deviations, $\sigma_{X_{1}}, \ldots, \sigma_{X_{n}}$. Assuming that its reference point is $k=\sum_{j} h_{j} k_{j}$, the degree of ambiguity of portfolio $Z=\sum_{j} h_{j} X_{j}$ is then

$$
\mho^{2}[Z]=4 \operatorname{Var}\left[\int_{-\infty}^{k} \frac{1}{\sqrt{2 \pi \sigma_{Z}^{2}}} e^{-\frac{\left(z-\mu_{Z}\right)^{2}}{2 \sigma_{Z}^{2}}} d z\right],
$$

where $\mu_{Z}=\sum_{j} h_{j} \mu_{X_{j}}$ is its random mean, and $\sigma_{Z}^{2}=\sum_{j} h_{j}^{2} \sigma_{X_{j}}^{2}+\sum_{j} \sum_{i \neq j} h_{j} h_{i} \rho_{X_{j}, X_{i}} \sigma_{X_{j}} \sigma_{X_{i}}$ is its random variance.

\footnotetext{
${ }^{14}$ To save on notations, an asset portfolio $Z$ is occasionally referred to by its return $Z$ rather than by its composition.
} 
This theorem allows us to inquire into the impact that the correlations among assets composing a portfolio have on its degree of ambiguity. The next theorem focuses on this relationship. Theorem 4. Let a portfolio $Z$ consist of $n$ assets whose returns are normally distributed. Assume nonnegative proportions $h_{1}, \ldots, h_{n}$ of assets in the portfolio, whose return $Z=\sum_{j} h_{j} X_{j}$ is characterized by the random mean $\mu_{Z}=\sum_{j} h_{j} \mu_{X_{j}}$ and the random variance $\sigma_{Z}^{2}=\sum_{j} h_{j}^{2} \sigma_{X_{j}}^{2}+$ $\sum_{j} \sum_{i \neq j} h_{j} h_{i} \rho_{X_{j}, X_{i}} \sigma_{X_{j}} \sigma_{X_{i}}$. If the reference point $k=\sum_{j} h_{j} k_{j}$ satisfies

$$
\mu_{Z}-\sigma_{Z} \sqrt{2} \leq k \leq \mu_{Z}+\sigma_{Z} \sqrt{2}
$$

for every $\mu_{Z}$ and $\sigma_{Z}$, then a higher correlation between any asset pair in $Z$ implies a lower degree of ambiguity of $Z$.

This theorem finds that, assuming a reference point within $\sqrt{2}$ standard deviations from the portfolio's means, the correlations among the assets composing this portfolio have a negative impact on its degree of ambiguity. At first glance, this result seems counterintuitive, since we are tempted to surmise that like risk, which increases with the correlations among assets, so too does ambiguity. However, this supposition is false. When the correlations among the returns on assets composing a portfolio are relatively high, the variance of its return is relatively high. By Theorem 2, the consequence of a higher variance is a lower degree of ambiguity.

The inverse relation between the correlations of returns and the degree of ambiguity holds as long as $\max \left(\mu_{Z}-\sigma_{Z} \sqrt{2}\right) \leq k \leq \min \left(\mu_{Z}+\sigma_{Z} \sqrt{2}\right)$. Reducing the correlations to a level sufficiently low while the reference point $k$ remains unchanged causes $k$ to violate Equation (15) by deviating from the range $\left[\max \left(\mu_{Z}-\sigma_{Z} \sqrt{2}\right), \min \left(\mu_{Z}+\sigma_{Z} \sqrt{2}\right)\right]$. In this case, lower correlations have a negative impact on the degree of ambiguity. The maximal possible correlation between any two assets $X_{i}$ and $X_{j}$ is $\rho_{X_{j}, X_{i}}=1$. Therefore, from Theorem 4 one can infer that any portfolio with assets having identical characteristics (mean and variance) is more ambiguous than each asset taken separately. The next theorem studies this property.

Theorem 5. Assume that the conditions of Theorem 4 hold and that $\frac{\mu_{X_{j}}}{\sigma_{X_{j}}}=\frac{\mu_{X_{i}}}{\sigma_{X_{i}}}, \forall X_{j}, X_{i}$. If short selling is prohibited then ambiguity is not diversifiable. The ambiguity of an asset portfolio is higher than the ambiguity each asset considered separately. That is,

$$
\mho^{2}\left[\sum_{j=1}^{n} h_{j} X_{j}\right] \geq \sum_{j=1}^{n} h_{j} \mho^{2}\left[X_{j}\right]=\mho^{2}[X],
$$

where for any $j=1, \ldots, n, \mho^{2}\left[X_{j}\right]=\mho^{2}[X]$ and $0 \leq h_{j}$, and $\sum_{j} h_{j}=1$. If short selling is allowed, then ambiguity can be diversified, but at the cost of higher risk 
By employing numerical simulations, one can show that the ability to diversify ambiguity is a matter of accounting for the relationships among the random means and random variances of returns. Figure 4, for example, simulates the degree of ambiguity of a two-asset portfolio as a function of the proportion of the assets in the portfolio. It considers two uncorrelated assets whose returns are normally distributed with known mean, $\mu_{X}=0.1$, random standard deviation that can be either 0.05 or 0.1 with equal likelihoods, and reference point $k=0.09$. The $\mathrm{x}$-axis shows the proportion of the first asset in the portfolio. The $\mathrm{y}$-axis shows the degree of ambiguity (solid line) and the degree of risk (dashed line) of the portfolio, where risk is measured by standard deviation. In this simulation one can see that the portfolio generates higher ambiguity as compared with the degree of ambiguity of each individual asset separately.

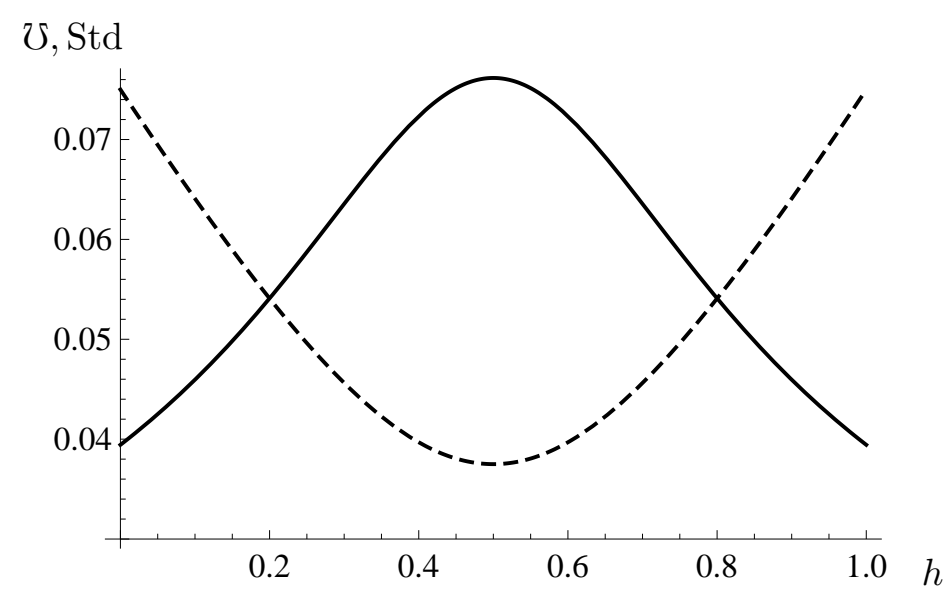

Figure 4: Ambiguity versus risk of a two-asset portfolio

This figure considers two uncorrelated assets whose returns are normally distributed with known mean, $\mu_{X}=0.1$, random standard deviation that can be either 0.05 or 0.1 with equal likelihoods, and reference point $k=0.09$. The $\mathrm{x}$-axis shows the proportion of the first asset in the portfolio. The y-axis shows the degree of ambiguity (solid line) and the degree of risk (dashed line) of the portfolio, where risk is measured by standard deviation.

This leads us to study the impact of the number of assets composing a portfolio on its degree of ambiguity.

Theorem 6. Assume an equally weighted portfolio $Z$ consisting of assets whose returns are normally distributed, all with identical random means and random variances. Let $\mu_{Z}$ and $\sigma_{Z}$ be the random mean and the random standard deviation of $Z$, respectively. If the reference point $k$ satisfies

$$
\mu_{Z}-\sigma_{Z} \sqrt{2} \leq k \leq \mu_{Z}+\sigma_{Z} \sqrt{2}
$$

for every $\mu_{Z}$ and $\sigma_{Z}$, adding an asset to the portfolio increases its degree of ambiguity while it 
decreases its degree of risk.

This theorem implies that as the number of (not perfectly positively correlated) assets in a portfolio tends to infinity, its variance tends to zero. In this case, one of two things can happen (if $k$ is not surrounded by $\mu_{X}$ ): either the reference point deviates by more than $\sqrt{2}$ standard deviations from the mean, or the reference point is equal to the mean. Both imply a perfectly diversifiable ambiguity, i.e., $\mho^{2}=0$. However, if $k$ is surrounded by $\mu_{X}$, the degree of ambiguity tends to its maximal possible value, 1 , as the variance tends to 0 . This is the case in reality, where different assets are characterized by different random means. As a result, portfolio composition might be concerned with the tradeoff between risk and ambiguity. In other words, investors "pay" for risk reduction by incurring higher ambiguity.

Figure 5 illustrates the impact of the number of assets composing a portfolio on its degree of ambiguity and its degree of risk. The figure considers uncorrelated assets whose returns are normally distributed with random mean that can be either 0.04 or 0.08 , and random variance that can be either 0.2 or 0.4 , all with equal likelihoods, and reference point $k=0.04$. The $\mathrm{x}$-axis shows the number of assets in the portfolio. The y-axis shows the degree of ambiguity (solid line) and the degree of risk (dashed line) of the portfolio, where risk is measured by standard deviation. It can be observed from this example that increasing the number of assets in the portfolio from 1 to 50 decreases its risk from 0.3 to almost 0 . Yet, it increases its degree of ambiguity from $\mho=0.04$ to $\mho=0.5$.

Our results have considerable implications for the conventional wisdom of the financial literature, which asserts that investors should minimize risk by holding a fully diversified portfolio. The implication is that when ambiguity is present, in most cases, holding such a portfolio is not optimal for ambiguity-averse investors, since for a given expected return, minimizing risk increases ambiguity. The precise composition of an optimal portfolio is determined subjectively by each investor according to her levels of risk aversion and ambiguity aversion. We leave the topic of optimal portfolio composition, reflective of investors' preferences, for future research.

The conclusions that emerge from our study coincide with field studies regarding individual investor behavior. These studies document that individuals tend to hold underdiversified portfolios (3-4 stocks); see for example Barber and Odean (2000) and Goetzmann and Kumar (2008). Bossaerts et al. (2010) demonstrate that ambiguity-averse investors are reluctant to hold ambiguous assets. In an earlier study Uppal and Wang (2003) demonstrate that a small difference in the ambiguity of the distribution of marginal returns results in a portfolio that is 


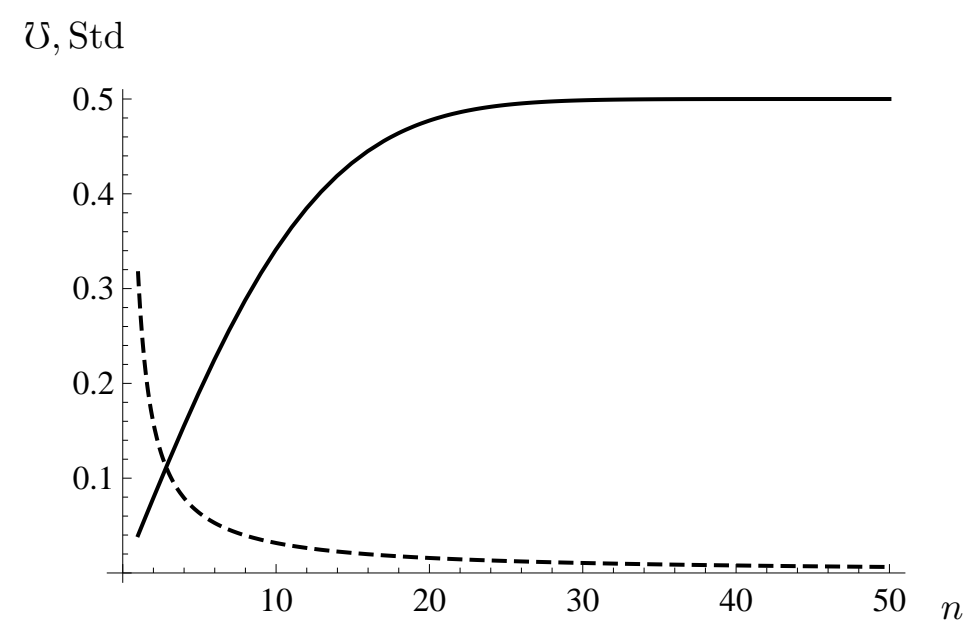

Figure 5: Ambiguity versus risk of an $\mathbf{n}$-asset portfolio

This figure considers uncorrelated assets whose returns are normally distributed with random mean that can be either 0.04 or 0.08 and random variance that can be either 0.2 or 0.4 , all with equal likelihoods, and reference point $k=0.04$. The x-axis shows the number of assets in the portfolio. The y-axis shows the degree of ambiguity (solid line) and the degree of risk (dashed line) of the portfolio, where risk is measured by standard deviation.

significantly underdiversified relative to the standard mean-variance portfolio. An explanation for these phenomena may look to the nature of ambiguity and its relationship with risk as presented in this paper. "Own-company stock," "home-country equity" and "limited stock market participation" are other financial puzzles upon which our model might help to shed new light.

\section{Conclusion}

This paper shows that it is not necessarily optimal to hold a fully diversified portfolio. The financial literature asserts that investors should minimize risk by diversifying their investments. This literature, however, assumes away ambiguity, the uncertainty over probabilities. When accounting for ambiguity one may falsely assume that as a portfolio is diversified for risk, it is also diversified for ambiguity. The present paper proves that risk minimization usually coincides with increased ambiguity, implying that holding a portfolio with minimal risk for a given expected return exposes investors to ambiguity that they may prefer not to bear.

The inverse relation between risk and ambiguity has significant implications for many aspects of financial decision making, especially concerning optimal portfolio selection. The optimal portfolios assembled by risk-averse and ambiguity-averse investors should minimize the aggregation of risk and ambiguity for a given expected return. Such a portfolio should take 
into account this inverse relation between risk and ambiguity and investors' attitudes toward each. As a result, investors may choose to hold nondiversified portfolios. The tradeoff between risk and ambiguity can shed new light on some puzzling financial phenomena. For example: underdiversification, where investors hold an average of only 3-4 stocks; limited market participation, where investors choose not to hold stocks at all; and the volatility risk premium, where investors overestimate the expected volatility of the stock market. 


\section{References}

Barber, B. M. and T. Odean (2000) "Trading Is Hazardous to Your Wealth: The Common Stock Investment Performance of Individual Investors," The Journal of Finance, Vol. 55, No. 2, pp. 773806.

Barberis, N. and M. Huang (2001) "Mental Accounting, Loss Aversion, and Individual Stock Returns," The Journal of Finance, Vol. 56, No. 4, pp. 1247-1292.

Bogan, V. (2008) "Stock Market Participation and the Internet," Journal of Financial and Quantitative Analysis, Vol. 43, No. 01, pp. 191-211.

Bollerslev, T., G. Tauchen, and H. Zhou (2009) "Expected Stock Returns and Variance Risk Premia," The Review of Financial Studies, Vol. 22, No. 11, pp. 4463-4492.

Bossaerts, P., P. Ghirardato, S. Guarnaschelli, and W. R. Zame (2010) "Ambiguity in Asset Markets: Theory and Experiment," The Review of Financial Studies, Vol. 23, No. 4, pp. 1325-1359.

Brenner, M. and Y. Izhakian (2011) "Asset Prices and Ambiguity: Empirical Evidance," Stern School of Business, Finance Working Paper Series, FIN-11-10.

Broadie, M., M. Chernov, and M. Johannes (2009) "Understanding Index Option Returns," The Review of Financial Studies, Vol. 22, pp. 4493-4529.

Cao, H. H., T. Wang, and H. H. Zhang (2005) "Model Uncertainty, Limited Market Participation, and Asset Prices," The Review of Financial Studies, Vol. 18, No. 4, pp. 1219-1251.

Carr, P. and L. Wu (2009) "Variance Risk Premiums," Review of Financial Studies, Vol. 22, No. 3, pp. $1311-1341$.

Coval, J. D. and T. J. Moskowitz (1999) "Home Bias at Home: Local Equity Preference in Domestic Portfolios," The Journal of Finance, Vol. 54, No. 6, pp. 2045-2073.

Dow, J. and S. R. d. C. Werlang (1992) "Uncertainty Aversion, Risk Aversion, and the Optimal Choice of Portfolio," Econometrica, Vol. 60, No. 1, pp. 197-204.

Drechsler, I. (2012) "Uncertainty, Time-Varying Fear, and Asset Prices," The Journal of Finance, Forthcoming.

Epstein, L. G. and T. Wang (1994) "Intertemporal Asset Pricing Under Knightian Uncertainty," Econometrica, Vol. 62, No. 2, pp. 283-322.

Eraker, B. (2004) "Do Stock Prices and Volatility Jump? Reconciling Evidence from Spot and Option Prices," The Journal of Finance, Vol. 59, No. 3, pp. 1367-1404.

Goetzmann, W. N. and A. Kumar (2008) "Equity Portfolio Diversification," Review of Finance, Vol. 12, No. 3, pp. 433-463.

Gollier, C. (2011) "Does Ambiguity Aversion Reinforce Risk Aversion? Applications to Portfolio Choices and Asset Pricing," The Review of Economic Stuies, Vol. 78, No. 4, pp. 1329-1344.

Guiso, L., P. Sapienza, and L. Zingales (2008) "Trusting the Stock Market," The Journal of Finance, Vol. 63, No. 6, pp. 2557-2600.

Guo, H. (2004) "Limited Stock Market Participation and Asset Prices in a Dynamic Economy," Journal of Financial and Quantitative Analysis, Vol. 39, No. 03, pp. 495-516.

Hirshleifer, D. (2001) "Investor Psychology and Asset Pricing," The Journal of Finance, Vol. 56, No. 4, pp. 1533-1597. 
Izhakian, Y. (2012a) "Ambiguity Measurement," Stern School of Business, Economics Working Paper Series, ECN-12-01.

(2012b) "Capital Asset Pricing under Ambiguity," Stern School of Business, Economics Working Paper Series, ECN-12-02.

Kothiyal, A., V. Spinu, and P. P.Wakker (2011) "Prospect Theory for Continuous Distributions: a Preference Foundation," Journal of Risk and Uncertainty, Vol. 42, No. 3, pp. 195-210.

Mehra, R. and E. C. Prescott (1985) "The Equity Premium: A Puzzle," Journal of Monetary Economics, Vol. 15, No. 2, pp. 145-161.

Mukerji, S. and J. M. Tallon (2001) "Ambiguity Aversion and Incompleteness of Financial Markets," The Review of Economic Studies, Vol. 68, No. 4, pp. pp. 883-904.

Schmeidler, D. (1989) "Subjective Probability and Expected Utility without Additivity," Econometrica, Vol. 57, No. 3, pp. 571-87.

Shiller, R. J. (1981) "Do Stock Prices Move Too Much to be Justified by Subsequent Changes in Dividends?" American Economic Review, Vol. 71, No. 3, pp. 421-36.

Todorov, V. (2010) "Variance Risk-Premium Dynamics: The Role of Jumps," The Review of Financial Studies, Vol. 23, No. 1, pp. 345-383.

Trojani, F. and P. Vanini (2004) "Robustness and Ambiguity Aversion in General Equilibrium," Review of Finance, Vol. 8, No. 2, pp. 279-324.

Tversky, A. and D. Kahneman (1992) "Advances in Prospect Theory: Cumulative Representation of Uncertainty," Journal of Risk and Uncertainty, Vol. 5, No. 4, pp. 297-323.

Ui, T. (2011) "The Ambiguity Premium vs. the Risk Premium under Limited Market Participation," Review of Finance, Vol. 15, No. 2, pp. 245-275.

Uppal, R. and T. Wang (2003) "Model Misspecification and Under Diversification," The Journal of Finance, Vol. 58, pp. 2465-2486.

Van Rooij, M., L. Annamaria, and A. Rob (2011) "Financial Literacy and Stock Market Participation," Journal of Financial Economics, Vol. 101, No. 2, pp. 449-472.

Wakker, P. (2010) Prospect Theory: For Risk and Ambiguity: Cambridge University Press.

Weil, P. (1989) "The Equity Premium Puzzle and The Risk-Free Rate Puzzle," Journal of Monetary Economics, Vol. 24, No. 3, pp. 401-421. 


\section{A Appendix}

\section{A.1 Supporting claims}

Lemma 1. Given an equally weighted portfolio consisting of assets whose returns have an identical mean $\mu_{X}$ and an identical variance $\sigma_{X}^{2}$, the variance of the portfolio's return decreases with the number of assets in the portfolio.

Theorem 7. Let $p_{x}=\mathrm{E}\left[\mathrm{P}_{x}\right]$ and $\zeta_{x}^{2}=\operatorname{Var}\left[\mathrm{P}_{x}\right]$, where $\mathrm{P}_{x}=\mathrm{P}(X \leq x)$ is the random probability of a random variable $X$, and assume a continuous twice-differentiable outlook function $\Gamma$, satisfying $\frac{1}{2}\left(\frac{\Gamma^{\prime \prime}\left(p_{x}\right)}{\Gamma^{\prime}\left(p_{x}\right)} \zeta_{x}^{2}-\frac{\Gamma^{\prime \prime}\left(p_{z}\right)}{\Gamma^{\prime}\left(p_{z}\right)} \zeta_{z}^{2}\right) \leq p_{z}$ for any $x \leq z \leq k$ and for any $k \leq z \leq x$. For a relatively small $\mathrm{P}_{x}$, the perceived probability of a random variable $X \leq x$ is then

$$
\mathrm{Q}(X \leq x)=\Gamma^{-1}\left(\int_{\mathbb{P}} \Gamma(\mathrm{P}(X \leq x)) d \chi(\mathrm{P})\right) \approx p_{x}+\frac{1}{2} \frac{\Gamma^{\prime \prime}\left(p_{x}\right)}{\Gamma^{\prime}\left(p_{x}\right)} \zeta_{x}^{2} .
$$

\section{A.2 Proofs}

Proof of Lemma 1. Let $Z$ be a portfolio consisting of $n$ assets. Its variance is

$$
\sigma_{Z}^{2}=\sum_{j=1}^{n} \frac{1}{n^{2}} \sigma_{X}^{2}+\sum_{j=1}^{n} \sum_{i \neq j} \sigma_{X}^{2} \rho
$$

where $-1 \leq \rho \leq 1$ is the correlation between each pair of assets in the portfolio. Simplifying this expression provides $\sigma_{Z}^{2}=\frac{1}{n} \sigma_{X}^{2}+\left(1-\frac{1}{n}\right) \sigma_{X}^{2} \rho$. Differentiating $\sigma_{Z}^{2}$ with respect to $n$ provides $\frac{\partial \sigma_{Z}^{2}}{\partial n}=-\frac{1}{n^{2}} \sigma_{X}^{2}+\frac{1}{n^{2}} \rho \sigma_{X}^{2} \leq 0$ for any $\rho$.

Proof of Proposition 1. Applying an increasing transformation $\mathrm{T}$ to all the outcomes $x$ of an asset $X$ does not change the ordering of these outcomes. The aggregate event of loss and its probabilities, $\mathrm{P}_{L}$, remain unchanged because the references point $k$ is adjusted accordingly. Therefore, the degree of ambiguity $\mho^{2}$ is not affected by the transformation $T$.

Proof of Proposition 2. Writing Var $[X]$ explicitly provides

$$
\begin{aligned}
\operatorname{Var}[X] & =\int_{\mathbb{P}}\left(\int_{\Omega}\left(X(\omega)-\int_{\mathbb{P}}\left(\int_{\Omega} X(\omega) d \mathrm{P}(\omega)\right) d \chi(\mathrm{P})\right)^{2} d \mathrm{P}(\omega)\right) d \chi(\mathrm{P}) \\
& =\int_{\mathbb{P}}\left(\int_{\Omega} X^{2}(\omega) d \mathrm{P}(\omega)\right) d \chi(\mathrm{P})-\left(\int_{\mathbb{P}}\left(\int_{\Omega} X(\omega) d \mathrm{P}(\omega)\right) d \chi(\mathrm{P})\right)^{2} .
\end{aligned}
$$

Since $\mu_{X}=\int_{\Omega} X(\omega) d \mathrm{P}(\omega)$, we can write

$$
\left(\int_{\mathbb{P}}\left(\int_{\Omega} X(\omega) d \mathrm{P}(\omega)\right) d \chi(\mathrm{P})\right)^{2}=\left(\int_{\mathbb{P}} \mu_{X} d \chi(\mathrm{P})\right)^{2}=\int_{\mathbb{P}} \mu_{X}^{2} d \chi(\mathrm{P})-\operatorname{Var}\left[\mu_{X}\right] .
$$


Substituting into Equation (20) yields

$$
\operatorname{Var}[X]=\int_{\mathbb{P}}\left(\int_{\Omega} X^{2}(\omega) d \mathrm{P}(\omega)\right) d \chi(\mathrm{P})-\int_{\mathbb{P}} \mu_{X}^{2} d \chi(\mathrm{P})+\operatorname{Var}\left[\mu_{X}\right]=\mathrm{E}\left[\sigma_{X}^{2}\right]+\operatorname{Var}\left[\mu_{X}\right]
$$

Proof of Corollary 1. Writing the ambiguity measure explicitly provides

$$
\mho^{2}[c+h X]=4 \operatorname{Var}\left[\mathrm{P}_{L}\right]=4 \operatorname{Var}\left[\int_{-\infty}^{c+h k} \frac{1}{\sqrt{2 \pi h^{2} \sigma_{X}^{2}}} e^{-\frac{\left(x-c-h \mu_{X}\right)^{2}}{2 h^{2} \sigma_{X}^{2}}} d x\right] .
$$

Changing the integration variable to $x=c+h y$ provides the required result.

Proof of Theorem 1. By Theorem 7, the expected utility of Equation (6) can be written

$$
\mathrm{V}(1+\mathrm{E}[Z]-\mathcal{K}) \approx-\int_{-\infty}^{k}\left[p_{z}+\frac{1}{2} \frac{\Gamma^{\prime \prime}\left(p_{z}\right)}{\Gamma^{\prime}\left(p_{z}\right)} \zeta_{z}^{2}\right] d z+\int_{k}^{\infty}\left[p_{z}+\frac{1}{2} \frac{\Gamma^{\prime \prime}\left(p_{z}\right)}{\Gamma^{\prime}\left(p_{z}\right)} \zeta_{z}^{2}\right] d z
$$

where $p_{z}=\mathrm{E}[\mathrm{P}(Z \leq z)]$ and $\zeta_{z}^{2}=\operatorname{Var}[\mathrm{P}(Z \leq z)]$. Changing the integration variable to $z=\mathrm{U}(1+x)$ provides

$$
\begin{aligned}
\mathrm{V}(1+\mathrm{E}[X]-\mathcal{K}) \approx & -\int_{-\infty}^{k}\left[p_{x}+\frac{1}{2} \frac{\Gamma^{\prime \prime}\left(p_{x}\right)}{\Gamma^{\prime}\left(p_{x}\right)} \zeta_{x}^{2}\right] \mathrm{U}^{\prime}(1+x) d x+ \\
& \int_{k}^{\infty}\left[1-p_{x}+\frac{1}{2} \frac{\Gamma^{\prime \prime}\left(1-p_{x}\right)}{\Gamma^{\prime}\left(1-p_{x}\right)} \zeta_{x}^{2}\right] \mathrm{U}^{\prime}(1+x) d x
\end{aligned}
$$

where $p_{x}=\mathrm{E}[\mathrm{P}(X \leq x)]$ and $\zeta_{x}^{2}=\operatorname{Var}[\mathrm{P}(X \leq x)]$. Integrating by parts provides

$$
\begin{aligned}
\mathrm{V}(1+\mathrm{E}[X]-\mathcal{K}) \approx & -\left.p_{x} \mathrm{U}(1+x)\right|_{-\infty} ^{k}+\int_{-\infty}^{k} \mathrm{E}\left[\phi\left(x ; \mu_{X}, \sigma_{X}\right)\right] \mathrm{U}(1+x) d x \\
& -\int_{-\infty}^{k} \frac{1}{2} \frac{\Gamma^{\prime \prime}\left(p_{x}\right)}{\Gamma^{\prime}\left(p_{x}\right)} \zeta_{x}^{2} \mathrm{U}^{\prime}(1+x) d x \\
& +\left.\left(1-p_{x}\right) \mathrm{U}(1+x)\right|_{k} ^{\infty}+\int_{k}^{\infty} \mathrm{E}\left[\phi\left(x ; \mu_{X}, \sigma_{X}\right)\right] \mathrm{U}(1+x) d x \\
& +\int_{k}^{\infty} \frac{1}{2} \frac{\Gamma^{\prime \prime}\left(1-p_{x}\right)}{\Gamma^{\prime}\left(1-p_{x}\right)} \zeta_{x}^{2} \mathrm{U}^{\prime}(1+x) d x
\end{aligned}
$$

where $\phi\left(x ; \mu_{X}, \sigma_{X}\right)$ stands for the normal probability density function. Because $\mathrm{U}(1+k)=0$, the sum of the first element in the first line and the first element in the third line in Equation (21) is zero. Since $k$ is relatively close to $\mathrm{E}[X]$, taking a first-order Taylor approximation (around $\mathrm{E}[X]$ ) of the second and the fourth lines of Equation (21) provides

$$
\begin{aligned}
I \approx & -\int_{-\infty}^{k} \frac{1}{2} \frac{\Gamma^{\prime \prime}\left(p_{k}\right)}{\Gamma^{\prime}\left(p_{k}\right)} \zeta_{k}^{2} \mathrm{U}^{\prime}(1+\mathrm{E}[X]) d x-\int_{-\infty}^{k} \frac{1}{2}\left[\frac{\Gamma^{\prime \prime}\left(p_{k}\right)}{\Gamma^{\prime}\left(p_{k}\right)} \zeta_{k}^{2} \mathrm{U}^{\prime}(1+\mathrm{E}[X])\right]^{\prime}(x-\mathrm{E}[X]) d x \\
& +\int_{k}^{\infty} \frac{1}{2} \frac{\Gamma^{\prime \prime}\left(1-p_{k}\right)}{\Gamma^{\prime}\left(1-p_{k}\right)} \zeta_{k}^{2} \mathrm{U}^{\prime}(1+\mathrm{E}[X]) d x+\int_{k}^{\infty} \frac{1}{2}\left[\frac{\Gamma^{\prime \prime}\left(1-p_{k}\right)}{\Gamma^{\prime}\left(1-p_{k}\right)} \zeta_{k}^{2} \mathrm{U}^{\prime}(1+\mathrm{E}[X])\right]^{\prime}(x-\mathrm{E}[X]) d x
\end{aligned}
$$

Thus, $I$ satisfies

$$
I \approx-\frac{1}{8} \frac{\Gamma^{\prime \prime}\left(p_{L}\right)}{\Gamma^{\prime}\left(p_{L}\right)} \mho^{2}[X] \mathrm{U}^{\prime}(1+\mathrm{E}[X])-\frac{1}{8} \frac{\Gamma^{\prime \prime}\left(p_{G}\right)}{\Gamma^{\prime}\left(p_{G}\right)} \mho^{2}[X] \mathrm{U}^{\prime}(1+\mathrm{E}[X]),
$$


where $p_{L}=\mathrm{E}\left[\mathrm{P}_{L}\right]$ and $p_{G}=\mathrm{E}\left[\mathrm{P}_{G}\right]$ are the expected probabilities of loss and gain, respectively. The second-order Taylor approximation (around $\mathrm{E}[X]$ ) of the second component in the first line and the second component in the third line of Equation (21) provides

$$
\begin{aligned}
I I & =\int_{-\infty}^{\infty} \mathrm{E}\left[\phi\left(x ; \mu_{X}, \sigma_{X}\right)\right] \mathrm{U}(1+x) d x \\
& \approx \int_{-\infty}^{\infty} \mathrm{E}\left[\phi\left(x ; \mu_{X}, \sigma_{X}\right)\right]\left(\begin{array}{l}
\mathrm{U}(1+\mathrm{E}[X])+\mathrm{U}^{\prime}(1+\mathrm{E}[X])(x-\mathrm{E}[X])+ \\
\frac{1}{2} \mathrm{U}^{\prime \prime}(1+\mathrm{E}[X])(x-\mathrm{E}[X])^{2}
\end{array}\right) d x \\
& =\mathrm{U}(1+\mathrm{E}[X])+\frac{1}{2} \mathrm{U}^{\prime \prime}(1+\mathrm{E}[X]) \operatorname{Var}[X] .
\end{aligned}
$$

The first-order Taylor approximation (around 0) of the LHS of Equation (21) with respect to $\mathcal{K}$ is

$$
L H S=\mathrm{U}(1+\mathrm{E}[X]-\mathcal{K}) \approx \mathrm{U}(1+\mathrm{E}[X])-\mathcal{K} \mathrm{U}^{\prime}(1+\mathrm{E}[X]) .
$$

Combining the LHS, $I$ and $I I$, the uncertainty premium is

$$
\mathcal{K} \approx-\frac{1}{2} \frac{\mathrm{U}^{\prime \prime}(1+\mathrm{E}[X])}{\mathrm{U}^{\prime}(1+\mathrm{E}[X])} \operatorname{Var}[X]-\frac{1}{8}\left[\frac{\Gamma^{\prime \prime}\left(p_{L}\right)}{\Gamma^{\prime}\left(p_{L}\right)}+\frac{\Gamma^{\prime \prime}\left(p_{G}\right)}{\Gamma^{\prime}\left(p_{G}\right)}\right] \mho^{2}[X] .
$$

Write the approximated expected utility as

$$
\mathrm{V}(1+\mathrm{E}[X]-\mathcal{K}) \approx \mathrm{U}\left(1+\mathrm{E}[X]+\frac{1}{2} \frac{\mathrm{U}^{\prime \prime}(1+\mathrm{E}[X])}{\mathrm{U}^{\prime}(1+\mathrm{E}[X])} \operatorname{Var}[X]+\frac{1}{8}\left[\frac{\Gamma^{\prime \prime}\left(p_{L}\right)}{\Gamma^{\prime}\left(p_{L}\right)}+\frac{\Gamma^{\prime \prime}\left(p_{G}\right)}{\Gamma^{\prime}\left(p_{G}\right)}\right] \mho^{2}[X]\right)
$$

Risk aversion implies $\frac{\mathrm{U}^{\prime \prime}}{\mathrm{U}^{\prime}}<0$, and ambiguity aversion implies $\frac{\Gamma^{\prime \prime}}{\Gamma^{\prime}}<0$. Since $\mathrm{U}$ is strictly increasing, a higher $\operatorname{Var}[X]$ or a higher $\mho^{2}[X]$, each imply a lower expected utility.

Proof of Theorem 2. Differentiating the degree of ambiguity, formed by Equation (8), with respect to $\sigma_{X}$ yields

$$
\frac{\partial \mho^{2}}{\partial \sigma_{X}}=2 \int_{\mathbb{P}}\left(\mathrm{P}-\int_{\mathbb{P}} \mathrm{P} d \chi(\mathrm{P})\right)\left(\mathrm{P}^{\prime}-\int_{\mathbb{P}} \mathrm{P}^{\prime} d \chi(\mathrm{P})\right) d \chi(\mathrm{P})=2 \mathrm{E}\left[(\mathrm{P}-\mathrm{E}[\mathrm{P}])\left(\mathrm{P}^{\prime}-\mathrm{E}\left[\mathrm{P}^{\prime}\right]\right)\right]
$$

where $\mathrm{P}^{\prime}=\frac{\partial \mathrm{P}}{\partial \sigma_{X}}$. By the Leibniz integral rule

$$
\mathrm{P}^{\prime}=\frac{\partial \mathrm{P}}{\partial \sigma_{X}}=-\frac{\left(k-\mu_{X}\right)}{\sigma_{X}^{2} \sqrt{2 \pi}} e^{-\frac{\left(k-\mu_{X}\right)^{2}}{2 \sigma_{X}^{2}}}
$$

If $k \leq \mu_{X}$, then $\mathrm{P}^{\prime} \geq 0$, and therefore $\mathrm{P}$ increases in $\sigma_{X}$. Otherwise, if $k>\mu_{X}$, then $\mathrm{P}$ decreases in $\sigma_{X}$. Next, we differentiate $\mathrm{P}^{\prime}$ with respect to $\sigma_{X}$ to find its slope:

$$
\frac{\partial \mathrm{P}^{\prime}}{\partial \sigma_{X}}=-\frac{\left(k-\mu_{X}\right)}{\sigma_{X}^{5} \sqrt{2 \pi}}\left[\left(k-\mu_{X}\right)^{2}-2 \sigma_{X}^{2}\right] e^{-\frac{\left(k-\mu_{X}\right)^{2}}{2 \sigma_{X}^{2}}} .
$$

If $k \leq \mu_{X}$, then $\frac{\partial \mathrm{P}^{\prime}}{\partial \sigma_{X}}$ is non-positive when $\left(k-\mu_{X}\right)^{2}-2 \sigma_{X}^{2} \leq 0$. That is, if $\mu_{X}-\sigma_{X} \sqrt{2} \leq k \leq \mu_{X}$, then $\frac{\partial \mathrm{P}^{\prime}}{\partial \sigma_{X}} \leq 0$. However, if $\mu_{X} \leq k \leq \mu_{X}+\sigma_{X} \sqrt{2}$, then $\frac{\partial \mathrm{P}^{\prime}}{\partial \sigma_{X}} \geq 0$. This implies that when 
$\mu_{X}-\sigma_{X} \sqrt{2} \leq k \leq \mu_{X}, \mathrm{P}^{\prime}$ is a decreasing function of $\sigma_{X}$; and when $\mu_{X} \leq k \leq \mu_{X}+\sigma_{X} \sqrt{2}, \mathrm{P}^{\prime}$ is an increasing function of $\sigma_{X}$. However, when $k \leq \mu_{X}, \mathrm{P}$ is an increasing function of $\sigma_{X}$; and when $k \geq \mu_{X}, \mathrm{P}$ is a decreasing function of $\sigma_{X}$. Therefore, the covariance between $\mathrm{P}$ and $\mathrm{P}^{\prime}$ (Equation (22)) is negative, which implies that $\frac{\partial \mho^{2}}{\partial \sigma_{X}} \leq 0$.

Proof of Theorem 3. Using the convolution theorem, it can be shown that the sum of normally distributed variables is also normally distributed with mean $\mu_{Z}=\sum_{j} h_{j} \mu_{X_{j}}$ and variance $\sigma_{Z}^{2}=\sum_{j} h_{j}^{2} \sigma_{X_{j}}^{2}+\sum_{j} \sum_{i \neq j} h_{j} h_{i} \rho_{X_{j}, X_{i}} \sigma_{X_{j}} \sigma_{X_{i}}$. Equation (8) then proves the theorem.

Proof of Theorem 4. Writing the ambiguity of $Z$ explicitly provides

$$
\begin{aligned}
\mho^{2}[Z] & =4 \operatorname{Var}\left[\int_{-\infty}^{k} \frac{1}{\sqrt{2 \pi \sigma_{Z}^{2}}} e^{-\frac{\left(z-\mu_{Z}\right)^{2}}{2 \sigma_{Z}^{2}}} d z\right] \\
& =4 \operatorname{Var}\left[\Phi\left(\frac{k-\sum_{j} h_{j} \mu_{X_{j}}}{\sqrt{\sum_{j} h_{j}^{2} \sigma_{X_{j}}^{2}+\sum_{j} \sum_{j \neq i} h_{j} h_{i} \rho_{X_{j}, X_{i}} \sigma_{X_{j}} \sigma_{X_{i}}}}\right)\right],
\end{aligned}
$$

where the second equality is obtained by changing the integration variables and substituting for $\mu_{Z}$ and $\sigma_{Z}$. Since any asset has a nonnegative weight in the portfolio, a higher correlation implies a greater $\sigma_{Z}$, which in turn, by Theorem 2, implies a lower degree of ambiguity.

Proof of Theorem 5. This theorem is proved for a two-asset portfolio consisting of assets $X$ and $Y$. The proof can then be extended by induction to any number of assets. Since $\frac{\mu_{X}}{\sigma_{X}}=\frac{\mu_{Y}}{\sigma_{Y}}$, then $\mu_{Y}=a \mu_{X}$ and $\sigma_{Y}=a \sigma_{X}$. Therefore, since $k$ is adjusted accordingly, the degree of ambiguity associated with each of the two assets is identical:

$$
4 \operatorname{Var}\left[\Phi\left(\frac{k_{Y}-\mu_{Y}}{\sigma_{Y}}\right)\right]=4 \operatorname{Var}\left[\Phi\left(\frac{a k_{X}-a \mu_{X}}{a \sigma_{X}}\right)\right]=4 \operatorname{Var}\left[\Phi\left(\frac{k_{X}-\mu_{X}}{\sigma_{X}}\right)\right] .
$$

Hence, $h \mho^{2}[X]+(1-h) \mho^{2}[Y]=4 \operatorname{Var}\left[\Phi\left(\frac{k-\mu_{X}}{\sigma_{X}}\right)\right]$, where $h$ and $(1-h)$ are the proportions of $X$ and $Y$ in the portfolio, respectively. The degree of ambiguity associated with the asset portfolio is

$$
\begin{aligned}
I & =4 \operatorname{Var}\left[\Phi\left(\frac{k-h \mu_{X}-(1-h) \mu_{Y}}{\sqrt{h^{2} \sigma_{X}^{2}+(1-h)^{2} \sigma_{Y}^{2}+2 h(1-h) \rho_{X, Y} \sigma_{X} \sigma_{Y}}}\right)\right] \\
& =4 \operatorname{Var}\left[\Phi\left(\frac{k-h \mu_{X}-a(1-h) \mu_{X}}{\sqrt{h^{2} \sigma_{X}^{2}+(1-h)^{2} a^{2} \sigma_{X}^{2}+2 a h(1-h) \rho_{X, Y} \sigma_{X}^{2}}}\right)\right],
\end{aligned}
$$

where $k=h k_{X}+(1-h) k_{Y}=h k_{X}+a(1-h) k_{X}$. When $0 \leq h \leq 1$, by Theorem 4 , $I$ attains 
its minimal value when the correlation is maximal, i.e., when $\rho_{X, Y}=1$; thus,

$$
I \geq 4 \operatorname{Var}\left[\Phi\left(\frac{h k_{X}+a(1-h) k_{X}-h \mu_{X}-a(1-h) \mu_{X}}{\sqrt{h^{2} \sigma_{X}^{2}+(1-h)^{2} a^{2} \sigma_{X}^{2}+2 a h(1-h) \sigma_{X}^{2}}}\right)\right]=4 \operatorname{Var}\left[\Phi\left(\frac{k_{X}-\mu_{X}}{\sigma_{X}}\right)\right] .
$$

When short selling is allowed, one can increase the variance of a portfolio by choosing some $h<0$. Then, by Theorem 2, the degree of ambiguity is lower. However, by Proposition 2, the perceived volatility (risk) is then higher.

Proof of Theorem 6. By Lemma 1, a greater number of assets in a portfolio implies a lower variance which, by Proposition 2, implies a lower perceived volatility (risk). Theorem 2 proves that a lower variance results in a higher degree of ambiguity.

Proof of Theorem 7. The LHS of Equation (18) can be written as

$$
\mathrm{Q}(X \leq x)=\Gamma^{-1}\left(\Gamma\left(p_{x}-\varphi\right)\right)=\Gamma^{-1}\left(\int_{\mathbb{P}} \Gamma\left(\mathrm{P}_{x}\right) d \chi(\mathrm{P})\right)
$$

for some $\varphi$, where $p_{x}=\mathrm{E}[\mathrm{P}(X \leq x)]$. Ignoring $\Gamma^{-1}$ and taking the first-order Taylor approximation of $\Gamma\left(p_{x}-\varphi\right)$ around 0 with respect to $\varphi$ yields

$$
\Gamma\left(p_{x}-\varphi\right) \approx \Gamma\left(p_{x}\right)+\Gamma^{\prime}\left(p_{x}\right)\left(p_{x}-\varphi-p_{x}\right)=\Gamma\left(p_{x}\right)-\varphi \Gamma^{\prime}\left(p_{x}\right) .
$$

Ignoring $\Gamma^{-1}$ and the integration in the RHS of Equation (24) for the moment, the second-order Taylor approximation of $\Gamma\left(\mathrm{P}_{x}\right)$ around $p_{x}$ is

$$
\Gamma\left(\mathrm{P}_{x}\right) \approx \Gamma\left(p_{x}\right)+\Gamma^{\prime}\left(p_{x}\right)\left(\mathrm{P}_{x}-p_{x}\right)+\frac{1}{2} \Gamma^{\prime \prime}\left(p_{x}\right)\left(\mathrm{P}_{x}-p_{x}\right)^{2} .
$$

Since $\Gamma\left(p_{x}\right), \Gamma^{\prime}\left(p_{x}\right)$ and $\Gamma^{\prime \prime}\left(p_{x}\right)$ are constants, applying the integration provides

$$
\int_{\mathbb{P}} \Gamma\left(\mathrm{P}_{x}\right) d \chi(\mathrm{P}) \approx \Gamma\left(p_{x}\right)+\frac{1}{2} \Gamma^{\prime \prime}\left(p_{x}\right) \zeta_{x}^{2}
$$

Equating $(25)$ to $(26)$ and rearranging terms yields $\varphi \approx-\frac{1}{2} \frac{\Gamma^{\prime \prime}\left(p_{x}\right)}{\Gamma^{\prime}\left(p_{x}\right)} \zeta_{x}^{2}$. Substituting $\varphi$ into Equation (24) proves the theorem. 